\title{
Erratum: Effect of first-forbidden decays on the shape of neutrino spectra [Phys. Rev. C 91, 025503 (2015)]
}

\author{
Dong-Liang Fang and B. Alex Brown \\ (Received 14 February 2016; published 15 April 2016)
}

DOI: 10.1103/PhysRevC.93.049903

In our paper we stated that "in [1] only the $M_{0}^{s}, u, x$, and $z$ terms were used for FF branches," and we discuss comparison with this "simplified operator." The authors of Ref. [1] have pointed out to us that their formalism is different from ours, and that the full operator is taken into account by the $J_{v}$ and $\rho_{A}$ terms of footnote 16 of Ref. [1]. Thus the curves labeled "Simp. Oper." in Figs. 2(a) and 2(b) in the paper do not represent the approximation used in Ref. [1]. As a consequence, our remarks about the difference between our results and those of Ref. [1] are not correct.

[1] A. C. Hayes, J. L. Friar, G. T. Garvey, G. Jungman, and G. Jonkmans, Phys. Rev. Lett. 112, 202501 (2014). 